\title{
Transition Zone Migration: A Mechanism for Cytoplasmic Ciliogenesis and Postaxonemal Centriole Elongation
}

\author{
Tomer Avidor-Reiss, Andrew Ha, and Marcus L. Basiri \\ University of Toledo, Department of Biological Sciences, Toledo, Ohio 43606 \\ Correspondence: tomer.avidorreiss@utoledo.edu
}

The cilium is an elongated and continuous structure that spans two major subcellular domains. The cytoplasmic domain contains a short centriole, which serves to nucleate the main projection of the cilium. This projection, known as the axoneme, remains separated from the cytoplasm by a specialized gatekeeping complex within a ciliary subdomain called the transition zone. In this way, the axoneme is compartmentalized. Intriguingly, however, this general principle of cilium biology is altered in the sperm cells of many animals, which instead contain a cytoplasmic axoneme domain. Here, we discuss the hypothesis that the formation of specialized sperm giant centrioles and cytoplasmic cilia is mediated by the migration of the transition zone from its typical location as part of a structure known as the annulus and examine the intrinsic properties of the transition zone that may facilitate its migratory behavior.

$T^{\mathrm{h}}$

his review specifically addresses a phenome-

non unique to certain ciliated cell types whereby a ciliary substructure known as the transition zone migrates distally from its original position between the centriole and the cilium during the process of axoneme assembly. We begin our discussion with a general introduction to cilium biology that is primarily intended for those who are less familiar with the transition zone and other fundamental ciliary substructures. Next, we build on this foundation by introducing the relatively recent observation that, in certain sperm cell types, the transition zone and a related structure known as the annulus migrates away from the centriole and its original position at the base of the ciliary compartment. We then consider the ways in which transition zone migration may contribute to centriole elongation, and we end our discussion by hy- pothesizing the mechanism by which transition zone migration enables cytoplasmic ciliogenesis.

\section{CENTRIOLE, CILIUM, AND TRANSITION ZONE}

Centriole, Centrosome, Basal Body, and Cilium Are Related Terms

The centriole lives a double life. In dividing cells, the centriole is located centrally, where it functions to organize and maintain dense layers of protein complexes referred to as pericentriolar material around its periphery to assemble the centrosome and participate in mitotic activities. Once mitosis ends, the centriole sheds the majority of its centrosomal complexes and travels to the cell membrane where it produces a cilium during interphase $\left(\mathrm{G}_{0 / 1}\right)$. If the cell cycle continues to mitosis, the cilium is disassembled and the

Editors: Wallace Marshall and Renata Basto

Additional Perspectives on Cilia available at www.cshperspectives.org

Copyright (C) 2017 Cold Spring Harbor Laboratory Press; all rights reserved; doi: 10.1101/cshperspect.a028142 Cite this article as Cold Spring Harb Perspect Biol 2017;9:a028142 
T. Avidor-Reiss et al.

centriole returns to the center of the cell where it again nucleates centrosomal complexes $\left(\mathrm{S}-\mathrm{G}_{2}\right)$. Therefore, the centrosome and cilium are distinct functional and morphological manifestations of the same organelle, shifting back and forth throughout the cell cycle to accomplish separate cellular responsibilities. Of note, the centriole is historically referred to as the "basal body" while it is a component of the cilium. For simplicity, we will use the term centriole in the context of both the centrosome and cilium.

\section{A Centriole and a Cilium Form a Continuous Microtubule-Based Structure Dynamic at One End}

In broad terms, the cilium is comprised of two parts: a cytoplasmic segment characterized by the centriole, and a second longer segment that projects from the cell surface via a structure known as the axoneme. Although, these ciliary substructures are often regarded separately, the axoneme is not structurally discrete from the centriole but is instead formed as a continuous extension of the centriole's distal end that gains compositional and functional distinction by incorporating axoneme-specific proteins (Fig. 1A). Importantly, a common and continuous polymer of microtubules forms both structures. At its proximal segment, the centriole is comprised of a ninefold radial array of triplet microtubules, by convention referred to as the "A,"
" $\mathrm{B}$," and " $\mathrm{C}$ " microtubules (Fig. 1A). This radial arrangement continues distally along the centriole and throughout the axoneme via extension of the " $\mathrm{A}$ " and "B" microtubules. Considering this relationship, the centriole can therefore be regarded as both the template and the cytoplasmic anchor for the axoneme.

Because microtubules have an inherent polarity, the centriole and axoneme are also polarized, with the microtubule "minus" end found at the proximal end of the centriole and the "plus" end found at the distal end of the axoneme. In general, microtubule minus ends are stabilized by complexes formed from $\gamma$-tubulin ( $\gamma$-TuRC) (Moritz et al. 1995). Consistently, it appears that the proximal end of centriolar "A" microtubules are also stabilized by a $\gamma$-TuRC complex (Guichard et al. 2010). On the other hand, however, microtubule "plus" ends are dynamic, displaying the capacity for both dramatic extension and collapse (Mitchison and Kirschner 1984; Desai and Mitchison 1997). Similarly, axonemal microtubules are highly dynamic at their growing "plus" ends, enabling them to form cilia of distinct lengths (Johnson and Rosenbaum 1992).

\section{Typical Centriole Elongation Precedes Axoneme Assembly}

Although the capacity for length variability is an inherent property of microtubule-based struc-

\footnotetext{
Figure 1. Models of transition zone migration in centriole formation and ciliogenesis. $(A)$ In a typical cell, the centriole forms in the cytoplasm. At its base, the centriole is comprised of triplet microtubules known as the A, B, and $\mathrm{C}$ microtubules, with only the $\mathrm{A}$ and $\mathrm{B}$ microtubules extending to its tip. The centriole then either associates with a vesicle that fuses with the plasma membrane (not shown) or migrates directly to the membrane for docking. There, the microtubules at the distal end of the centriole elongate to form an axoneme, which is surrounded by a specialized membrane known as the ciliary membrane. At the base of the cilium, this membrane forms a pocket known as the ciliary pocket, and the axoneme is embedded in a network of proteins that serves as a ciliary gate known as the transition zone. (B) During Drosophila spermatogenesis, ciliogenesis begins in the premeiotic diploid spermatocytes and is completed in the postmeiotic haploid spermatids. In the spermatocyte, the centriole docks to the plasma membrane and forms a short cilium. After the axoneme is initiated, both the centriole and the cilium elongate. Presumably, the centriole grows as a result of transition zone migration along the axoneme. In the spermatid, the cilium continues to grow and the transition zone continues to migrate along the axoneme, exposing axonemal microtubules to the cytoplasm. $(C)$ During mammalian spermatogenesis, ciliogenesis starts and is completed in spermatids. In the spermatid, the centriole docks to the plasma membrane and forms a full-length cilium. After the cilium is formed, the transition zone and annulus migrate along the axoneme. Proteins involved in transition zone migration and annulus formation and migration are indicated in $B$ and $C$.
} 

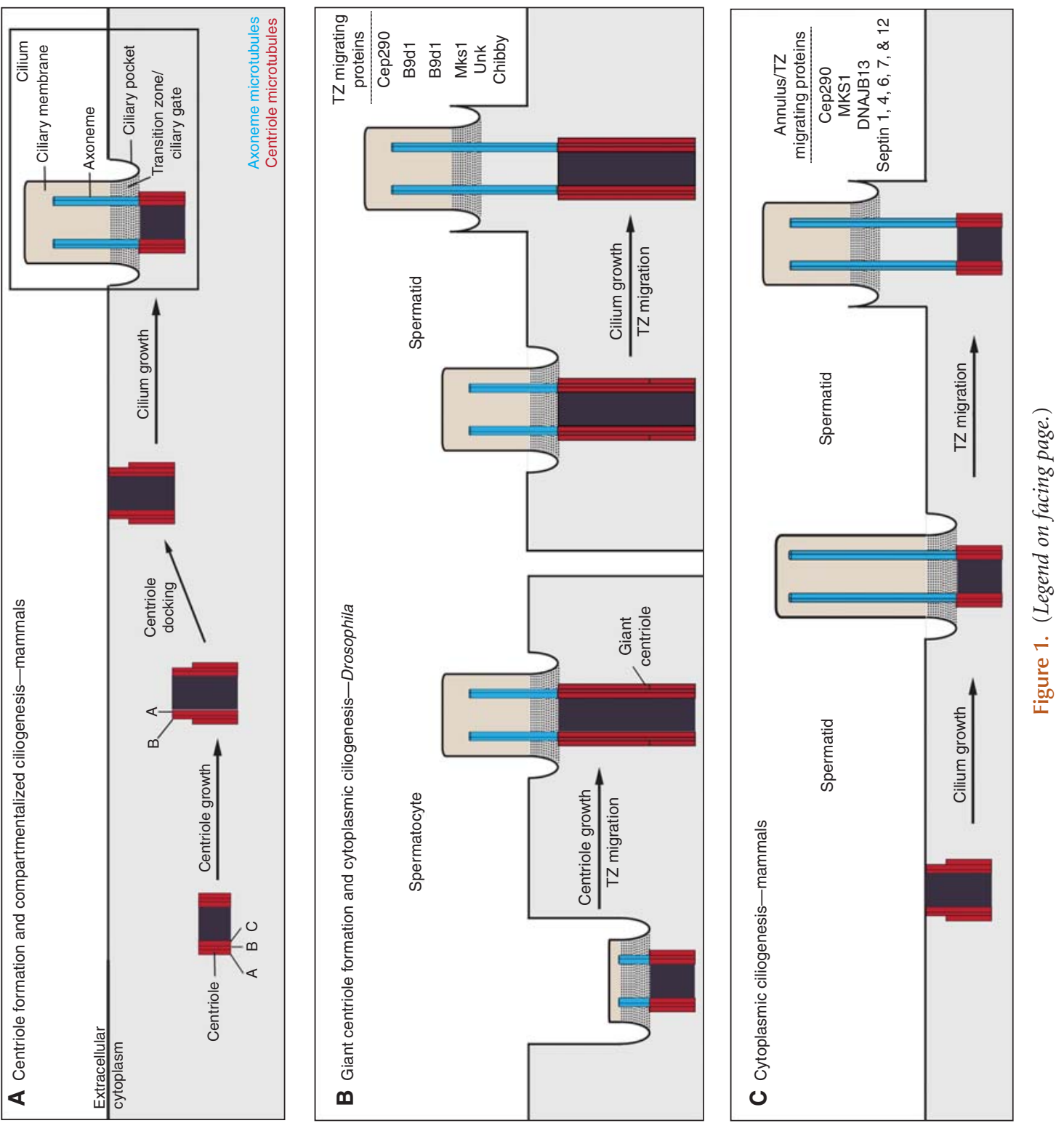
tures, centriole length is strictly regulated, with precise lengths consistently observed within similar settings. Still, this characteristic length can vary between contexts and is dependent on the organism, cell type, and stage of the cell cycle. Centriole elongation occurs via a regulated program that proceeds before axoneme assembly. For example, mature mammalian centrioles typically measure $\sim 500 \mathrm{~nm}$ in length, whereas centrioles of Drosophila early embryos are $\sim 200 \mathrm{~nm}$ long (Gonzalez et al. 1998). In mammals, a centriole reaches its final length in a stepwise process occurring across almost two cell cycles. Initially, an $\sim 200$-nm-long procentriole is made from a centriolar scaffold called the cartwheel during $\mathrm{S}$ phase. The centriole continues to elongate throughout $\mathrm{G}_{2}$ and $\mathrm{M}$ to reach $\sim 500 \mathrm{~nm}$ in length. Typically, the conclusion of centriole elongation is marked by the incorporation of distal appendage proteins. Once a cell with a mature centriole is committed for ciliogenesis, the centriole's distal " $A$ " and " $B$ " microtubules elongate to form the axoneme. In most cells, centriole length is thought to become locked following the onset of axoneme assembly, remaining stable not only throughout subsequent phases of ciliogenesis but also throughout the life of the mature functional cilium. Therefore, to summarize, centriole length is tightly controlled, displaying little variability within its specific biological context, and final centriole length is usually achieved before the initiation of axoneme assembly.

\section{The Typical Cilium Is Compartmentalized by a Transition Zone}

As described above, the axoneme can be regarded as an uninterrupted extension of the microtubule-based architecture of the centriole, upon which is layered a unique and defining complement of axoneme-specific proteins. Thus, various mechanisms must ensure that the centriole and axoneme faithfully establish and maintain their distinct compositions, and therefore functions. One such mechanism is an inherent consequence of the stepwise assembly process directing centriole and axoneme growth. As the centriole grows distally, centriolar proteins are initially deposited on the microtubule scaffold. This process is similarly mirrored during axoneme assembly, with the deposition of axonemal proteins occurring instead. This differential deposition occurring at the level of the centriole and axoneme is intimately tied to the activity of both a ciliary trafficking mechanism known as intraflagellar transport (IFT) (Cole et al. 1998; Rosenbaum and Witman 2002) and a molecular gatekeeping complex within a structure known as the transition zone, which resides at the interface of the cytoplasmic centriole and the axonemal compartment (Craige et al. 2010; Garcia-Gonzalo et al. 2011). Together, IFT and the transition zone ciliary gate cooperate to maintain the centriole and axoneme within distinct cellular compartments. The spatial separation of the centriole and axoneme encourages the differential deposition of specific proteins onto otherwise structurally similar substrates, and axoneme compartmentalization is generally thought to be an essential prerequisite for normal ciliogenesis.

In a typical cilium, the transition zone is found just distal to the centriole and represents the most proximal segment of the cilium. Within this region, the axoneme becomes anchored to the membrane of the cilium, which takes on a characteristic bend known as the ciliary pocket (Fig. 1A). The transition zone is composed of a complex arrangement of both cytoplasmic and membrane-bound proteins that are thought to both connect the axoneme to the base of the ciliary membrane and to establish an active sieve that gates ciliary entry and exit at both the level of the cytoplasm and membrane (GarciaGonzalo et al. 2011). As a result of its multiple activities, the transition zone is compositionally diverse. For example, mass spectrometry of the centrosome-cilium interface using proximity-dependent biotinylation and capture has recently shown that at least 22 distinct proteins may comprise the mammalian transition zone (Gupta et al. 2015). In addition to cilium-specific proteins, the transition zone also contains multiple proteins of the Septin family (Chih et al. 2012), members of the nuclear import machinery such as Ran-GTP, importins and nucleoporins (Kee et al. 2012), and ESCRT pro- 
teins (Diener et al. 2015). To add to this complexity, the transition zone functions as an intricate network, with extensive protein-protein interactions occurring both within the transition zone and with outside partners (Sang et al. 2011; Williams et al. 2011). Nevertheless, although the various roles of individual transition zone proteins remain poorly understood, proteomic and genetic studies have shown that transition zone proteins segregate into distinct functional modules defined by core interacting partners and related mutant phenotypes. Accordingly, these modules have been named by the clinical manifestations associated with mutations of their respective componentsnamely, nephronopthisis (NPHP module) and Meckel-Gruber syndrome (MKS module) (Sang et al. 2011; Williams et al. 2011). Still, it remains unclear which components of the transition zone are either directly or indirectly involved in axoneme compartmentalization.

Besides its interactions with the axoneme and ciliary membrane, the transition zone is also intimately associated with the distal end of the centriole. For example, just proximal to the transition zone, centriolar structures known as the distal appendages are involved in attaching the centriole to the cell membrane and docking various complexes for ciliary transport (Deane et al. 2001). Furthermore, transition zone proteins also directly interact with components of the main centriole body (Gupta et al. 2015). Thus, the transition zone is generally regarded as both spatially anchored to and functionally dependent on the distal end of the centriole (Wang et al. 2013). Accordingly, like centriole elongation, transition zone formation generally occurs before axoneme formation, thereby ensuring that axoneme assembly occurs entirely within a separate cellular compartment.

\section{Sperm Cells Have Specialized Centrioles and Cilia}

Until now, we have discussed generalized features of ciliogenesis that occur in most species and cell types. To briefly reiterate, we described that centriole elongation occurs before the ini- tiation of axoneme assembly and that final centriole length is usually $\leq 500 \mu \mathrm{m}$. Next, we discussed that axoneme assembly occurs entirely within a separate cellular compartment protected by a gatekeeping mechanism within the transition zone. Intriguingly, however, sperm cells-which are motile by means of a modified cilium (often referred to as the flagellum) - often infringe on these general rules of cilium biology, showing two important distinctions: (1) the centriole is longer and (2) a portion of the axoneme is exposed to the cytoplasm (Fig. 2 ). These unique centrioles, which occur in some insect species and in non-passerine birds, can reach $2-5 \mu \mathrm{m}$ in length (up to 10 times typical centriole length) and are referred to as giant centrioles. Also, unlike typical fully compartmentalized cilia, the proximal segment of the axoneme in such species is exposed to the cytoplasm (Fig. 1). Nevertheless, electron microscopy studies have shown that in these species, the distal end of the cilium still forms what appears to be a typical membrane-bound axoneme (Fig. 2B).

Together, these observations have led to the distinction between two broad types of cilia, which are classified as either "cytoplasmic" or "compartmentalized" (Avidor-Reiss et al. 2004; Avidor-Reiss and Leroux 2015). Although this unique ciliary phenomena occurring in spermatids has been observed for many decades, little is known about how or why spermatid ciliogenesis forms giant centrioles and cytoplasmic axonemes. However, one adaptation that appears to be central to these processes is the disengagement of the transition zone from the centriole and its successive distal migration throughout axoneme elongation (Basiri et al. 2014).

\section{CENTRIOLE ELONGATION AND TRANSITION ZONE MIGRATION}

\section{Giant Centrioles Are Found In Sperm Cells of Some Insects}

Giant centrioles were originally described in insects using electron microscopy, first by Friedländer and Wahrman (1966) in neuropteran meiosis and shortly after by Phillips (1967) in 
T. Avidor-Reiss et al.
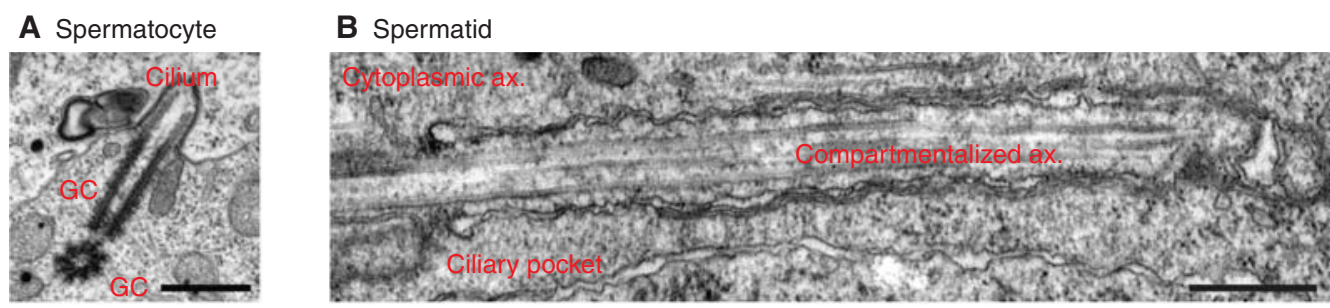

C Early spermatid

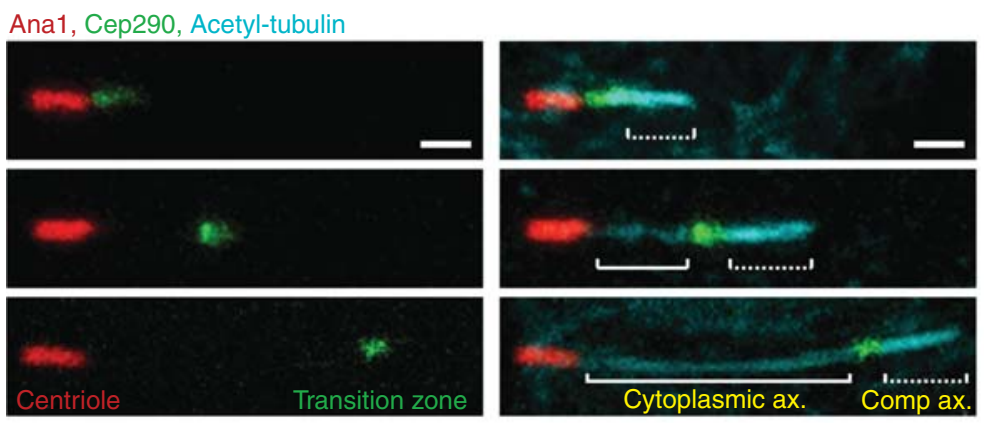

D Intermediate spermatid

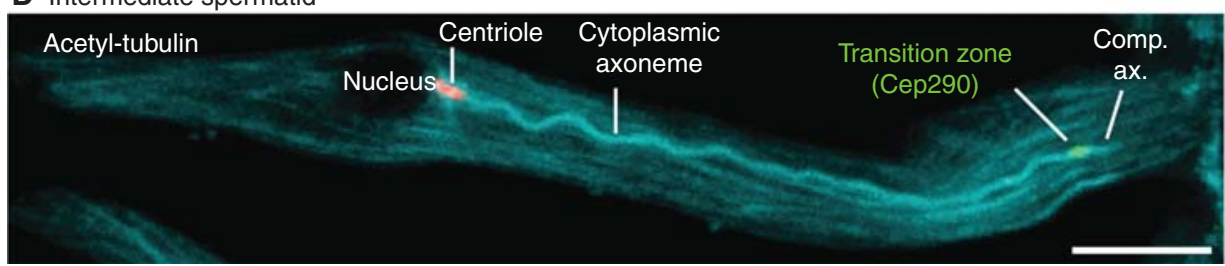

Figure 2. Transition zone migration in Drosophila melanogaster. (A) Electron micrograph showing two giant centrioles, one of which forms a short cilium. Scale bar, $500 \mathrm{~nm}$. (B) Electron micrograph showing a spermatid cytoplasmic and compartmentalized axoneme segment. The ciliary pocket is observed at the base of the compartmentalized axoneme. Scale bar, $500 \mathrm{~nm}$. (C) Light microscopy demonstrating transition zone migration in early spermatids. The centriole is labeled by Anal-tdTomato (red), transition zone is labeled by Cep290GFP (green), and axoneme (ax) is labeled with antiacetylated-tubulin (cyan). (D) A full-length intermediate spermatid with centriole, cytoplasmic axoneme, transition zone, and compartmentalized axoneme. ( $D$, From Basiri et al. 2014; reprinted, with permission, from Elsevier (C) 2014.)

sperm cells of Sciara. Later, atypically long centrioles were found in other insects including Drosophila melanogaster (Tates 1971), in which they were studied in the most detail. In Drosophila, during early spermatogenesis (from stem cells until early spermatocytes), mitotic centrioles display the typical short length representative of other tissue types (Tates 1971). Shortly afterward, these short centrioles dock to the plasma membrane and form a cilium. However, unlike most cell types in which centriole elongation terminates on attaching to the membrane, the centrioles of Drosophila spermatocytes and early spermatids unexpectedly continue to elongate extensively after membrane attachment and axoneme nucleation. During this stage of elongation, the centriole grows fourfold, ultimately reaching a length of $\sim 1.8 \mu \mathrm{m}$ while attached to the plasma membrane (Tates 1971; Basiri et al. 2013). Simultaneously, the ciliary axoneme slowly begins to grow from the distal end of the centriole to provide a platform for transition zone assembly. This modest axoneme growth occurs through- 
out most of the spermatocyte differentiation program and does not extend beyond the region defined by transition zone markers at the level of light microscopy (Tates 1971; Riparbelli et al. 2013; Basiri et al. 2014).

\section{Transition Zone Migration in Drosophila Spermatocytes May Enable Postaxonemal Centriole Elongation}

Typically, the transition zone is regarded as a static structure whose position does not change after its assembly. However, a static model of the transition zone poses problems for postaxonemal centriole elongation. So, how can a centriole elongate after forming a cilium? Theoretically, there are three possibilities: by elongating at its microtubule "plus" end, its "minus" end, or at some point within. Based on our current understanding of the rules governing microtubule assembly, it is unlikely that centrioles elongate via the latter two options. Considering the synonymous microtubulebased scaffolding of both the centriole and the axoneme as well as the capacity of the transition zone to migrate, it is tempting to speculate that distal displacement of the transition zone would expose bare, immature axoneme to the cytoplasm where it would be transformed into a centriole. Such a transformation could be accomplished by incorporating centriolar proteins to the freshly exposed " $A$ " and " $\mathrm{B}$ " microtubules of the axoneme and concurrent extension of centriolar " $\mathrm{C}$ " microtubules (Fig. 1C).

\section{Giant Centrioles Are Also Present in Some Vertebrate Taxa}

Similar to Drosophila and other insect species, the centrioles of some non-passerine birds are also considerably longer than in most species and cell types. In fact, in ostrich, the sperm distal centriole elongates to become giant in the spermatid stage, outgrowing Drosophila giant centrioles to ultimately reach $3 \mu \mathrm{m}$ in length (Soley 1994). However, there are important distinctions between Drosophila and ostrich giant centrioles. For example, unlike Drosophila in which spermatid giant centrioles are present in the background of cytoplasmic ciliogenesis, ostrich giant centrioles occur during an otherwise typical compartmentalized ciliogenesis. Furthermore, the distal centriole in ostrich grows to its final "giant" length before docking to the plasma membrane, and no centriole elongation occurs after the initiation of axoneme formation (Soley 1994). Thus, giant centrioles can also form outside of cytoplasmic ciliogenesis and transition zone migration, although in such cases final giant centriole length must be achieved before membrane docking.

\section{CYTOPLASMIC CILIOGENESIS AND TRANSITION ZONE MIGRATION}

\section{The Axoneme Is Exposed to the Cytoplasm in Some Cells}

Although a general theme of cilium biology is that the axoneme resides within a distinct compartment isolated from the cytoplasm via the transition zone, the exclusion of the axoneme from the cytoplasm is not an absolute requirement for ciliogenesis and examples of cytoplasmic axonemes are not uncommon (AvidorReiss and Leroux 2015). These "cytoplasmic cilia" can occur via three different mechanisms. Primary cytoplasmic ciliogenesis is found in mammalian sperm cells and is characterized by the exposure of the mature axoneme to the cytoplasm only after it is first fully formed within a compartmentalized cilium. In secondary cytoplasmic ciliogenesis, which is found in insect sperm cells, immature axoneme is exposed to the cytoplasm after its initial polymerization within a small compartment referred to as the ciliary cap. Tertiary cytoplasmic ciliogenesis is found in the microgametes of some protists such as plasmodium and is characterized by both the assembly and maturation of the axoneme in the cytoplasm. In all three types of cytoplasmic ciliogenesis, the axoneme eventually becomes associated with the cell membrane to form a tight and slender structure according to cell-type-specific processes.

In primary and secondary cytoplasmic ciliogenesis, the exposure of the axoneme to the cytoplasm requires the displacement of the at- 
tachment point between the axoneme and the ciliary membrane. This membrane attachment occurs at an electron-dense structure, known as the annulus in mammals (Fawcett et al. 1970) or the ring centriole in insects (Phillips 1970). For clarification, please note that the ring centriole is not a true microtubule-based centriole, but instead only resembles a centriole by its location at the base of a compartmentalized cilium. Similar to the transition zone of typical compartmentalized cilia, the annulus is essential for compartmentalizing the ciliary membrane by preventing exchange with the cell membrane (Caudron and Barral 2009). Recently, the annulus and ring centriole have been shown to contain typical transition zone components such as Septins and MKS module proteins (Ihara et al. 2005; Basiri et al. 2014).

Transition Zone Migration in D. melanogaster Spermatids Forms the Cytoplasmic Cilium

Earlier, we described the modest axoneme elongation that occurs in the context of giant centriole growth and transition zone assembly in Drosophila spermatocytes. However, in Drosophila, axoneme growth is not initiated in earnest until the conclusion of meiosis. Here, axoneme assembly involves a dramatic elongation program in which axoneme growth occurs in parallel to spermatid morphological differentiation, ultimately yielding an axoneme that is $\sim 2 \mathrm{~mm}$ in length. This exaggerated spermatid length seems to be the manifestation of evolutionarily pressures favoring increased spermatid length (Joly et al. 2004). Interestingly, although this extreme axoneme growth is required for the formation of motile spermatids, spermatid elongation occurs independently of axoneme assembly and appears to be driven by a mitochondrial mechanism (Noguchi et al. 2011). Transmission electron microscopy of Drosophila spermatids performed in the 1970s were the first to show that in this context of dramatic axoneme growth, the point of association of the ciliary membrane with the axoneme becomes displaced distally, ultimately exposing the majority of the axoneme to the cytoplasm
(Tates 1971; Tokuyasu 1975). Although this region of axoneme membrane association contains morphological features reminiscent of a typical compartmentalized cilium, the axoneme it encloses is structurally and compositionally immature (Fig. 2B) and only represents the final $2 \mu \mathrm{m}$ of what will eventually become an $\sim 2$-mm-long cytoplasmic axoneme.

In Drosophila spermatids, axoneme elongation is accompanied by transition zone disengagement from the distal end of the centriole. As the axoneme is assembled, the transition zone continuously migrates in close association with the axoneme's growing end. Throughout this process, the migrating transition zone remains consistently localized $\sim 2 \mu \mathrm{m}$ proximal to the very tip of the axoneme and remains coupled to the base of the ciliary pocket, which is pulled distally in concert with spermatid elongation. Thus, the migrating transition zone of Drosophila spermatids is perfectly situated to compartmentalize a cilium-like structure housing the growing axoneme tip. This unique centriole-detached structure, which is referred to as the ciliary cap, seems to contain the minimum elements required for axoneme compartmentalization. For example, immunostaining Drosophila spermatids with anti-acetyl-tubulin under a protocol that selectively permeabilizes the cell membrane without disrupting membrane of the ciliary cap allows for labeling of the entire cytoplasmic segment of the axoneme while excluding any labeling inside of the ciliary cap. Consistently, mutations of transition zone proteins such as Cep290 compromise the integrity of the ciliary cap compartment, allowing for staining of both the cytoplasmic and ciliary cap axoneme segments following selective permeabilization of the cell membrane without disrupting the ciliary cap membrane. Interestingly, mutation of Drosophila Cep290 also disrupts spermatid axoneme assembly, suggesting that compartmentalization of the site of axoneme assembly within the ciliary cap is essential for proper ciliogenesis in this system (Basiri et al. 2014).

In this context, two simultaneous and discrete processes characterize axoneme assembly 
in Drosophila spermatids. Within the ciliary cap compartment distal to the transition zone, axonemal microtubules are extended via patterned tubulin polymerization, the template for which was originally established in the centriole (Riparbelli et al. 2013). As the transition zone migrates, it leaves behind bare axonemal microtubules assembled in the appropriate architecture. Simultaneously, as this "naked" axoneme becomes exposed to the cytoplasm, axoneme-specific proteins become incorporated into its structure, representing the second phase of axoneme assembly (Tates 1971; Tokuyasu 1974). These proteins, which include dyneins and other axonemal proteins, are essential for sperm tail function in motility. Thus, axoneme assembly during spermatid cytoplasmic ciliogenesis occurs via compartmentalized polymerization and subsequent cytoplasmic maturation, with the migrating transition zone separating the two processes.

\section{The Annulus Is a Septin-Based Ring Structure} Found in Mammalian Spermatozoa

The mammalian sperm tail is structurally divided into four parts from proximal to distal: the connecting piece, midpiece, principal piece, and end piece. The connecting piece contains the centriole as well as other specialized structures such as a modified PCM assembly known as the striated columns. In some instances, the midpiece contains a cytoplasmic axoneme segment and mitochondria (Phillips 1974); we refer to this arrangement as an "axonemal midpiece" (Fig. 3A). However, in many fish and non-passerine bird species, the connecting piece and the midpiece are combined and contain the centriole, striated columns, and mitochondria without a cytoplasmic axoneme segment (Mattei 1988; Soley 1994), an arrangement that we refer to as a "centriolar midpiece" (Fig. 3A). Apart from this distinction, however, the junction of the midpiece and principal piece in most vertebrate species is morphologically similar, and is marked by a distinct ciliary pocket with a ring-like annulus (Fig. 3B,C). Thus, the base of the midpiece resembles the base of a typical compartmentalized cilium.
The annulus was originally characterized ultrastructurally as comprised of closely packed filaments attached to the membrane of the ciliary pocket (Fig. 3C) (Fawcett 1970). More recently, molecular studies have shown that the annulus contains several members of the Septin family of polymerizing GTP-binding proteins including Septin 1, 4, 6, 7, and 12 (Ihara et al. 2005; Steels et al. 2007). Although septin family proteins play diverse roles in many cell types, they have been shown to be particularly essential for spermatid development and motility. For example, loss-of-function mutations in Septin 12 and Septin 4 abolish the annulus and result in a marked kink at the midpieceprincipal piece junction in spermatozoa, resulting in male infertility in both mice and humans (Kuo et al. 2012). Despite these morphological defects, however, these mutants displayed a fully formed midpiece (Ihara et al. 2005; Kissel et al. 2005; Kwitny et al. 2010), suggesting that the annulus is dispensable for structurally delineating the midpiece-principal piece boundary. Still, Septin 4 knockout mice show defects in the compartmentalization of membrane proteins in the midpiece, suggesting that the annulus functions as a membrane diffusion barrier to prevent the exchange of membrane components between the midpiece and principal piece (Kwitny et al. 2010). Thus, the annulus displays a similar structural and functional role to the transition zone in compartmentalizing the distal ciliary membrane.

\section{Annulus Migration Happens during Spermiogenesis in Mammals and Some Birds during Flagellum Formation}

In many vertebrates, the annulus is formed during the early stages of spermiogenesis after the centrioles dock to the plasma membrane, but before the axoneme initiates its assembly (Holstein and Roosen-Runge 1981). Initially, the annulus is located at the distal end of the centriole, later departing from this position and migrating in concert with axoneme growth. Annulus migration then stops at a precise location that is species-specific and remains in this position in the mature sperm. As a result of this migration, 
A

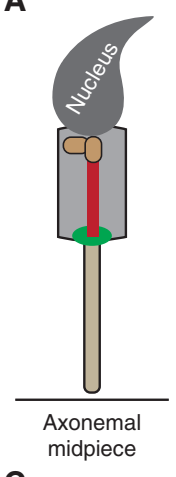

C

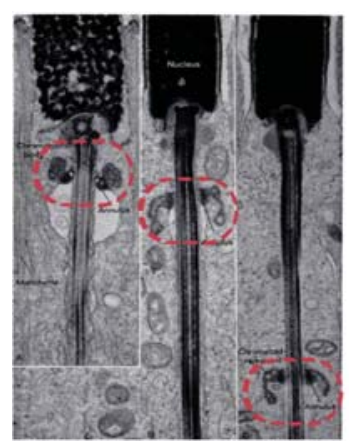

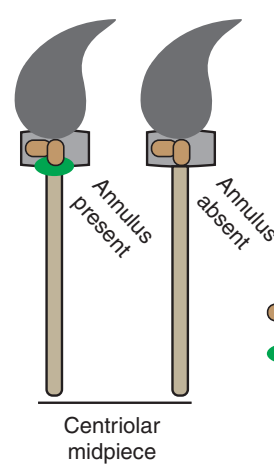

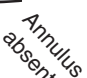

(1)

Comp. axoneme

Cyto. axoneme

D

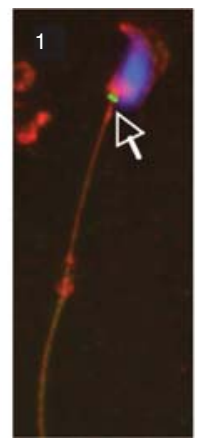

B

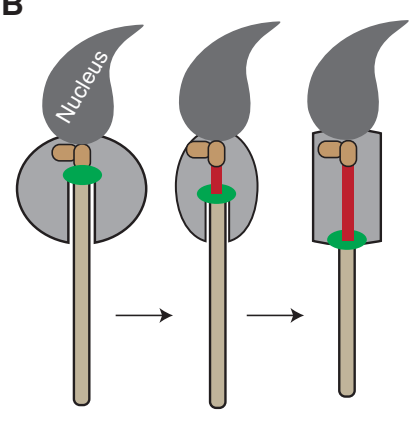

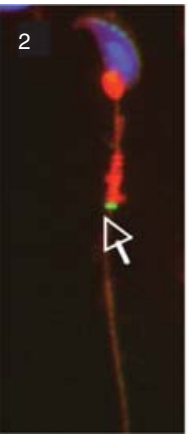

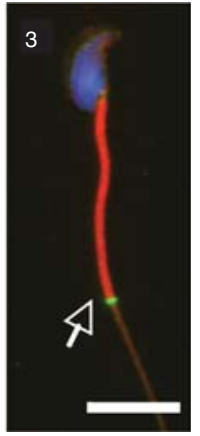

$\mathbf{E}$

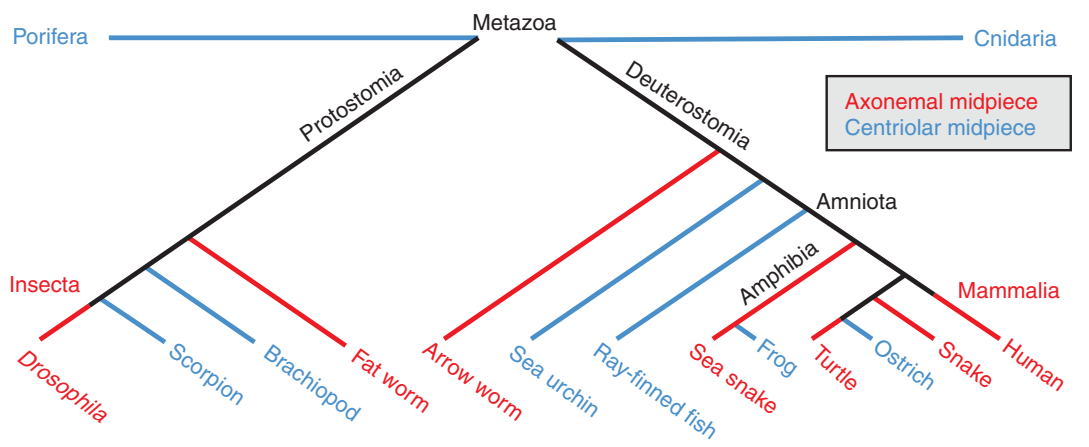

Figure 3. Annulus migration occurs in mammalian spermatids. (A) Sperm can be classified into three types based on the location and presence of the annulus: sperm with an annulus separating cytoplasmic and compartmentalized axoneme segments (axonemal midpiece, left), sperm with an annulus separating the centriole from a fully compartmentalized axoneme (centriolar midpiece with annulus), and sperm lacking an annulus but containing a centriolar midpiece and a fully compartmentalized axoneme within the principal piece (centriolar midpiece without annulus). (B) Illustration of annulus migration in mammalian spermatids. $(C)$ Longitudinal sections of Macaca mulatta spermatids demonstrating annulus migration during spermiogenesis. The annulus is indicated by a red dotted circle. (C, Reprinted from data in Fawcett et al. 1970.) (D) Septin 4 localization during mouse spermiogenesis (white arrow). The annulus indicated by Septin 4 antibody (green) is found near the nucleus (blue) in early stage elongating spermatids (1). The annulus then begins to migrate toward the growing end of the axoneme to form the midpiece (red) (2). Finally, the annulus reaches the distal end of the midpiece to define the midpiece-principal piece junction in mature sperm (3). ( $D$, From Guan et al. 2009; reprinted under the terms of the Creative Commons Attribution License.) Scale bar, $10 \mu \mathrm{m}$. (E) Distribution of spermatids containing either a centriolar midpiece or an axonemal midpiece across animal phylogeny. (Data based on Afzelius 1955; Silveira and Porter 1964; Sato et al. 1967; Reger and Cooper 1968; Mattei 1988; Hess et al. 1991; Mita and Nakamura 1992; Medina 1994; Dallai et al. 1995; Iomini and Justine 1997; Reunov and Klepal 2003; Scheltinga et al. 2003; Smita et al. 2004; Al-Dokhi et al. 2007, 2010; Vignoli et al. 2008; Lipke et al. 2009.) 
the midpiece contains both the centriole and a cytoplasmic segment of axoneme of defined length (Fig. 3C) (Nagano 1962; Fawcett et al. 1970; Phillips 1974; Gunawardana and Scott 1977). In mice, this process of annulus migration is nicely shown by the marker Septin 4 (Fig. 3D) (Guan et al. 2009). Aside from Septins, the annulus has also been shown to be marked by the co-chaperone DNAJB13 (Guan et al. 2009) in a manner independent of Septin 4 during early spermiogenesis, as well as the SLC26 family protein testis anion transporter 1 (Tat1) in mature sperm (Toure et al. 2007).

In some animals, such as the primitive nonpasserine birds, ostrich and rhea, the annulus does not migrate but instead maintains a fixed position throughout spermiogenesis (Soley 1994). In such cases, the annulus is formed when the centriole pair docks to the plasma membrane, ultimately creating a midpiece that is only composed of a centriole without a cytoplasmic axoneme segment (centriolar midpiece) (Fig. 3A) (Soley 1994). Considering this, the annulus is likely to have a role that is independent of transition zone migration.

Surprisingly, there are also animals in which electron microscopy does not identify an annulus. In these cases, the midpiece is only composed of a centriole, similar to spermatozoa in which the annulus maintains a fixed position (Jamieson et al. 1995; Lovas et al. 2012). Although untested, it is possible that these spermatids do in fact contain an annulus-like septinbased structure that is not apparent via electron microscopy.

Considering their similarities, it is likely that the migrating transition zone (the ring centrioles of insects) and the annulus are distinct names for the same ciliary structure. Both structures migrate away from the centriole during axoneme growth (Guan et al. 2009; Basiri et al. 2014). Furthermore, both contain transition zone proteins and are essential for compartmentalizing the two homologous segments of the sperm, namely, the Drosophila ciliary cap and the vertebrate sperm principle piece (Kissel et al. 2005; Basiri et al. 2014). One important distinction, however, is the timing of migration; annulus migration occurs after cilium forma- tion, whereas transition zone migration in Drosophila occurs during axoneme growth. It remains untested whether the Drosophila migrating transition zone also contains a septinbased component that may contribute to ciliary cap compartmentalization.

\section{Transition Zone Migration May Be} Motor-Driven or Exchange-Driven

The mechanism of transition zone migration has not yet been characterized. Because migration occurs along the axoneme, one can imagine that microtubule-based motors are involved. Alternatively, migration may occur via a treadmilling mechanism in which the transition zone is disassembled at the "minus" end and reassembled at the "plus" end.

Studies on the ciliary microtubule-binding protein Cep162 may provide insight into the mechanisms of migration. In RPE1 cells, siRNA depletion of Cep162 (QN1/KIAA1009), which normally localizes at the centriole just beyond the distal appendages, was shown to prevent the recruitment of various transition zone proteins, thereby blocking the initiation of ciliogenesis (Wang et al. 2013). Interestingly, expression of mutant forms of Cep162 that are unable to bind the distal centriole but with intact axoneme binding capacity was shown to result in the distal accumulation of transition zone proteins including Cep290, TMEM67, TCTN1, and RPGRIP1L at the ciliary tip. Cep162 interacts with Cep290 via its axoneme recognition domain, and although Cep290 can independently associate with the distal centriole, its microtubule binding capacity in mitotic centrosomal complexes is dependent on Cep162. Thus, the Cep290-Cep162 interaction appears to be required for its transition zone association with axoneme microtubules, and untethering the transition zone from the distal centriole may permit an inherent constitutive transition zone migratory capacity to exert itself.

Cep162 is an ATPase that contains structural similarity with the kinesin microtubule plusend motor family (Leon et al. 2006). Because this domain was not disrupted in the centriolebinding mutant (Wang et al. 2013), it is tempt- 
T. Avidor-Reiss et al.

ing to speculate that untethering Cep162 and, therefore indirectly, the transition zone, may allow for active transition zone migration via a Cep162 kinesin-type activity. Interestingly, the Drosophila ortholog of Cep162 (CG42699, via BLAST reciprocal best-hit) (data not shown) is enriched in the Drosophila testis (Chintapalli et al. 2007), and multiple sequence alignment of mammalian Cep162 and CG42699 (using Clustal Omega) (data not shown) shows that Drosophila does not seem to contain the amino-terminal region Cep162 that is essential for centriole binding in mammals (Wang et al. 2013). Nevertheless, aberrant displacement of the transition zone to the distal axoneme tip has not been reported in mutations of any other ciliary protein in compartmentalized cilia.

On the other hand, one can also speculate that transition zone migration occurs not via a motorized active mechanism but instead by successive rounds of displacement and replacement on the growing axonemal microtubules in a "treadmilling" manner. Consistent with this, previous studies have shown that transition zone proteins are in a dynamic equilibrium with a cytoplasmic reserve, rapidly cycling in between transition zone and cytoplasmic fractions (Craige et al. 2010). In this way, diffusion of fixed transition zone complexes back into the cytoplasm could allow for the reintegration of new transition zone proteins at more distal locations on the growing axoneme.

\section{Transition Zone Migration May Reflect an Inherent Elasticity in the Ciliary Gate}

Investigation of animals from distinct phylogenic groups that possess flagellated sperm reveals that spermiogenesis always begins with a centriole and an immature compartmentalized cilium. However, sperm development ultimately forms either an axonemal midpiece or a centriolar midpiece. Of these, spermatozoa with a centriolar midpiece are considered to represent the ancestral (primitive) form in animal evolution (Baccetti 1982). Interestingly, however, a review of the literature reveals that phylogenetic clades often contain species of both types, with some bearing a centriolar midpiece and others an axonemal midpiece (Fig. 3E). Stated differently, the appearance and divergence of the axonemal midpiece from the primitive centriolar type does not occur at a single clear bifurcation in metazoan evolutionary history, but instead occurs seemingly indiscriminately and in manifold throughout deuterostome and protostome phylogeny. This observation suggests that transition zone migration can be acquired quickly via minor evolutionary changes, and that the capacity for migration is an intrinsic feature of spermatid cilia.

In light of this capacity, we speculate that transition zone migration may reflect an inherent elasticity in the ciliary gate that may be necessary to maintain cilium compartmentalization under external forces, even in traditional fully compartmentalized cilia. In Drosophila spermatids, mitochondria grow dramatically in the cytoplasm and provide the mechanical force required for spermatid elongation (Noguchi et al. 2011). As the mitochondria elongate, they advance the cell membrane in the direction of axoneme growth. Because the transition zone is anchored to the advancing cell membrane, the ciliary gate may adapt to these forces by migrating relative to the axoneme. However, forces impacting the transition zone are not exclusive to insect spermatids. Because cilia project from cell surfaces and are often motile, they are inherently exposed to significant internal and external forces. For example, in mammalian primary cilia exposed to fluid flow, force is predicted to be highest at the ciliary base where the transition zone is localized (Rydholm et al. 2010; Young et al. 2012). Therefore, even in compartmentalized cilia, the ciliary gate is expected to be elastic and show a dynamic association with the surrounding ciliary architecture to adapt to stresses that would otherwise compromise the ciliary compartment.

\section{ACKNOWLEDGMENTS}

This work is supported by Grant 1121176 (Division of Molecular and Cellular Biosciences [MCB]) from the National Science Foundation and R01GM098394 from the National Institute of General Medical Sciences. 


\section{REFERENCES}

Afzelius BA. 1955. The fine structure of the sea urchin spermatozoa as revealed by the electron microscope. $Z$ Zellforsch Mikrosk Anat 42: 134-148.

Al-Dokhi O, Al-Onazee Y, Mubarak M. 2007. Fine structure of the epididymal sperm of the snake Eryx jayakari (Squamata, Reptilia). Int J Zool Res 3: 1-13.

Avidor-Reiss T, Leroux MR. 2015. Shared and distinct mechanisms of compartmentalized and cytoplasmic ciliogenesis. Curr Biol 25: R1143-R1150.

Avidor-Reiss T, Maer AM, Koundakjian E, Polyanovsky A Keil T, Subramaniam S, Zuker CS. 2004. Decoding cilia function: Defining specialized genes required for compartmentalized cilia biogenesis. Cell 117: 527-539.

Baccetti B. 1982. The evolution of the sperm tail. Symp Soc Exp Biol 35: 521-532.

Basiri ML, Blachon S, Chim YC, Avidor-Reiss T. 2013. Imaging centrosomes in fly testes. J Vis Exp e50938.

Basiri ML, Ha A, Chadha A, Clark NM, Polyanovsky A, Cook B, Avidor-Reiss T. 2014. A migrating ciliary gate compartmentalizes the site of axoneme assembly in Drosophila spermatids. Curr Biol 24: 2622-2631.

Caudron F, Barral Y. 2009. Septins and the lateral compartmentalization of eukaryotic membranes. Dev Cell 16: 493-506.

Chih B, Liu P, Chinn Y, Chalouni C, Komuves LG, Hass PE, Sandoval W, Peterson AS. 2012. A ciliopathy complex at the transition zone protects the cilia as a privileged membrane domain. Nat Cell Biol 14: 61-72.

Chintapalli VR, Wang J, Dow JA. 2007. Using FlyAtlas to identify better Drosophila melanogaster models of human disease. Nat Genet 39: 715-720.

Cole DG, Diener DR, Himelblau AL, Beech PL, Fuster JC, Rosenbaum JL. 1998. Chlamydomonas kinesin-II-dependent intraflagellar transport (IFT): IFT particles contain proteins required for ciliary assembly in Caenorhabditis elegans sensory neurons. J Cell Biol 141: 993-1008.

Craige B, Tsao CC, Diener DR, Hou Y, Lechtreck KF, Rosenbaum JL, Witman GB. 2010. CEP290 tethers flagellar transition zone microtubules to the membrane and regulates flagellar protein content. J Cell Biol 190: 927-940.

Dallai R, Afzelius BA, Witalinski W. 1995. The axoneme of the spider spermatozoon. Boll Zool 62: 335-338.

Dallai R, Mercati D, Bu Y, Yin YW, Callaini G, Riparbelli MG. 2010. The spermatogenesis and sperm structure of Acerentomon microrhinus (Protura, Hexapoda) with considerations on the phylogenetic position of the taxon. Zoomorphology 129: 61-80.

Deane JA, Cole DG, Seeley ES, Diener DR, Rosenbaum JL. 2001. Localization of intraflagellar transport protein IFT52 identifies basal body transitional fibers as the docking site for IFT particles. Curr Biol 11: 1586-1590.

Desai A, Mitchison TJ. 1997. Microtubule polymerization dynamics. Annu Rev Cell Dev Biol 13: 83-117.

Diener DR, Lupetti P, Rosenbaum JL. 2015. Proteomic analysis of isolated ciliary transition zones reveals the presence of ESCRT proteins. Curr Biol 25: 379-384.

Fawcett DW. 1970. A comparative view of sperm ultrastructure. Biol Reprod 2: 90-127.
Fawcett DW, Eddy EM, Phillips DM. 1970. Observations on the fine structure and relationships of the chromatoid body in mammalian spermatogenesis. Biol Reprod 2: 129-153.

Friedlander M, Wahrman J. 1966. Giant centrioles in neuropteran meiosis. J Cell Sci 1: 129-144.

Garcia-Gonzalo FR, Corbit KC, Sirerol-Piquer MS, Ramaswami G, Otto EA, Noriega TR, Seol AD, Robinson JF Bennett CL, Josifova DJ, et al. 2011. A transition zone complex regulates mammalian ciliogenesis and ciliary membrane composition. Nat Genet 43: 776-784.

Gonzalez C, Tavosanis G, Mollinari C. 1998. Centrosomes and microtubule organisation during Drosophila development. J Cell Sci 111: 2697-2706.

Guan J, Kinoshita M, Yuan L. 2009. Spatiotemporal association of DNAJB13 with the annulus during mouse sperm flagellum development. BMC Dev Biol 9: 23.

Guichard P, Chretien D, Marco S, Tassin AM. 2010. Procentriole assembly revealed by cryo-electron tomography. EMBO J 29: 1565-1572.

Gunawardana VK, Scott MG. 1977. Ultrastructural studies on the differentiation of spermatids in the domestic fowl. J Anat 124: 741-755.

Gupta GD, Coyaud E, Goncalves J, Mojarad BA, Liu Y, Wu Q, Gheiratmand L, Comartin D, Tkach JM, Cheung SW, et al. 2015. A dynamic protein interaction landscape of the human centrosome-cilium interface. Cell 163: 1484-1499.

Hess RA, Thurston RJ, Gist DH. 1991. Ultrastructure of the turtle spermatozoon. Anat Rec 229: 473-481.

Holstein AF, Roosen-Runge EC. 1981. Atlas of human spermatogenesis. Grosse, Berlin.

Ihara M, Kinoshita A, Yamada S, Tanaka H, Tanigaki A, Kitano A, Goto M, Okubo K, Nishiyama H, Ogawa O, et al. 2005. Cortical organization by the septin cytoskeleton is essential for structural and mechanical integrity of mammalian spermatozoa. Dev Cell 8: 343-352.

Iomini C, Justine JL. 1997. Spermiogenesis and spermatozoon of Echinostoma caproni (Platyhelminthes, Digenea): Transmission and scanning electron microscopy, and tubulin immunocytochemistry. Tissue Cell 29: 107-118.

Jamieson BG, Koehler L, Todd BJ. 1995. Spermatozoal ultrastructure in three species of parrots (aves, Psittaciformes) and its phylogenetic implications. Anat Rec 241: 461-468.

Johnson KA, Rosenbaum JL. 1992. Polarity of flagellar assembly in Chlamydomonas. J Cell Biol 119: 1605-1611.

Joly D, Korol A, Nevo E. 2004. Sperm size evolution in Drosophila: Inter- and intraspecific analysis. Genetica 120: $233-244$.

Kee HL, Dishinger JF, Blasius TL, Liu CJ, Margolis B, Verhey KJ. 2012. A size-exclusion permeability barrier and nucleoporins characterize a ciliary pore complex that regulates transport into cilia. Nat Cell Biol 14: 431-437.

Kissel H, Georgescu MM, Larisch S, Manova K, Hunnicutt GR, Steller H. 2005. The Sept4 septin locus is required for sperm terminal differentiation in mice. Dev Cell 8: 353-364.

Kuo YC, Lin YH, Chen HI, Wang YY, Chiou YW, Lin HH, Pan HA, Wu CM, Su SM, Hsu CC, et al. 2012. SEPT12 
T. Avidor-Reiss et al.

mutations cause male infertility with defective sperm annulus. Hum Mutat 33: 710-719.

Kwitny S, Klaus AV, Hunnicutt GR. 2010. The annulus of the mouse sperm tail is required to establish a membrane diffusion barrier that is engaged during the late steps of spermiogenesis. Biol Reprod 82: 669-678.

Leon A, Omri B, Gely A, Klein C, Crisanti P. 2006. QN1/ KIAA1009: A new essential protein for chromosome segregation and mitotic spindle assembly. Oncogene 25: 1887-1895.

Lipke C, Meinecke-Tillmann S, Meyer W, Meinecke B. 2009. Preparation and ultrastructure of spermatozoa from green poison frogs, Dendrobates auratus, following hormonal induced spermiation (Amphibia, Anura, Dendrobatidae). Anim Reprod Sci 113: 177-186.

Lovas EM, Filippich LJ, Johnston SD. 2012. Spermiogenesis in the Australian cockatiel Nymphicus hollandicus. J Morphol 273: 1291-1305.

Mattei X. 1988. The flagellar apparatus of spermatozoa in fish. Ultrastructure and evolution. Biol Cell 63: 151-158.

Medina A. 1994. Spermiogenesis and sperm structure in the shrimp Parapenaeus longirostris (Crustacea: Dendrobranchiata): Comparative aspects among decapods. Marine Biol 119: 449-460.

Mita M, Nakamura M. 1992. Ultrastructural study of an endogenous energy substrate in spermatozoa of the sea urchin Hemicentrotus pulcherrimus. Biol Bull 182: 298 304.

Mitchison T, Kirschner M. 1984. Dynamic instability of microtubule growth. Nature 312: 237-242.

Moritz M, Braunfeld MB, Sedat JW, Alberts B, Agard DA. 1995. Microtubule nucleation by $\gamma$-tubulin-containing rings in the centrosome. Nature 378: 638-640.

Nagano T. 1962. Observations on the fine structure of the developing spermatid in the domestic chicken. J Cell Biol 14: 193-205.

Noguchi T, Koizumi M, Hayashi S. 2011. Sustained elongation of sperm tail promoted by local remodeling of giant mitochondria in Drosophila. Curr Biol 21: 805-814.

Phillips DM. 1967. Giant centriole formation in Sciara. J Cell Biol 33: 73-92.

Phillips DM. 1970. Insect sperm: Their structure and morphogenesis. J Cell Biol 44: 243-277.

Phillips DM. 1974. Spermiogenesis. Academic, New York.

Reger JF, Cooper DP. 1968. Studies on the fine structure of spermatids and spermatozoa from the millipede Polydesmus sp. J Ultrastruct Res 23: 60-70.

Reunov A, Klepal W. 2003. Ultrastructural study of spermatogenesis in Phoronopsis harmeri (Lophophorata, Phoronida). Helgol Mar Res 58: 1-10.

Riparbelli MG, Cabrera OA, Callaini G, Megraw TL. 2013. Unique properties of Drosophila spermatocyte primary cilia. Biol Open 2: 1137-1147.

Rosenbaum JL, Witman GB. 2002. Intraflagellar transport. Nat Rev Mol Cell Biol 3: 813-825.

Rydholm S, Zwartz G, Kowalewski JM, Kamali-Zare P, Frisk T, Brismar H. 2010. Mechanical properties of primary cilia regulate the response to fluid flow. Am J Physiol Renal Physiol 298: F1096-1102.
Sang L, Miller JJ, Corbit KC, Giles RH, Brauer MJ, Otto EA, Baye LM, Wen X, Scales SJ, Kwong M, et al. 2011. Mapping the NPHP-JBTS-MKS protein network reveals ciliopathy disease genes and pathways. Cell 145: 513-528.

Sato M, Oh M, Sakodas K. 1967. Electron microscopic study of spermatogenesis in the lung fluke (Paragonimus miyazakii). Z Zellforsch Mikrosk Anat 77: 232-243.

Scheltinga DM, Wilkinson M, Jamieson BG, Oommen OV. 2003. Ultrastructure of the mature spermatozoa of caecilians (Amphibia: Gymnophiona). J Morphol 258: 179192.

Silveira M, Porter KR. 1964. The spermatozoids of flatworms and their microtubular systems. Protoplasma 59: 240-265.

Smita M, George JM, Girija R, Akbarsha MA, Oommen OV. 2004. Spermiogenesis in caecilians Ichthyophis tricolor and Uraeotyphlus cf. narayani (Amphibia: Gymnophiona): Analysis by light and transmission electron microscopy. J Morphol 262: 484-499.

Soley JT. 1994. Centriole development and formation of the flagellum during spermiogenesis in the ostrich (Struthio camelus). J Anat 185: 301-313.

Steels JD, Estey MP, Froese CD, Reynaud D, Pace-Asciak C, Trimble WS. 2007. Sept12 is a component of the mammalian sperm tail annulus. Cell Motil Cytoskeleton 64: 794-807.

Tates AD. 1971. Cytodifferentiation during spermatogenesis in Drosophila melanogaster: An electron microscope study. Rijksuniversiteit de Leiden, Leiden, Netherlands.

Tokuyasu KT. 1974. Dynamics of spermiogenesis in Drosophila melanogaster. IV: Nuclear transformation. J Ultrastruct Res 48: 284-303.

Tokuyasu KT. 1975. Dynamics of spermiogenesis in Drosophila melanogaster. VI: Significance of "onion" nebenkern formation. J Ultrastruct Res 53: 93-112.

Toure A, Lhuillier P, Gossen JA, Kuil CW, Lhote D, Jegou B, Escalier D, Gacon G. 2007. The testis anion transporter 1 (Slc26a8) is required for sperm terminal differentiation and male fertility in the mouse. Hum Mol Genet 16: 1783-1793.

Vignoli V, Klann AE, Michalik P. 2008. Spermatozoa and sperm packages of the European troglophylous scorpion Belisarius xambeui Simon, 1879 (Troglotayosicidae, Scorpiones). Tissue Cell 40: 411-416.

Wang WJ, Tay HG, Soni R, Perumal GS, Goll MG, Macaluso FP, Asara JM, Amack JD, Tsou MF. 2013. CEP162 is an axoneme-recognition protein promoting ciliary transition zone assembly at the cilia base. Nat Cell Biol 15: 591-601.

Williams CL, Li C, Kida K, Inglis PN, Mohan S, Semenec L, Bialas NJ, Stupay RM, Chen N, Blacque OE, et al. 2011. MKS and NPHP modules cooperate to establish basal body/transition zone membrane associations and ciliary gate function during ciliogenesis. J Cell Biol 192: 10231041.

Young YN, Downs M, Jacobs CR. 2012. Dynamics of the primary cilium in shear flow. Biophys J 103: 629-639. 


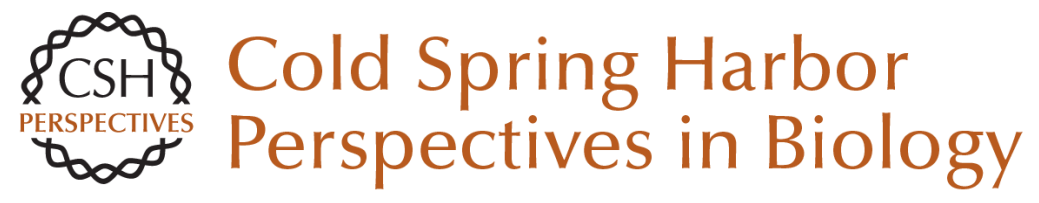

\section{Transition Zone Migration: A Mechanism for Cytoplasmic Ciliogenesis and Postaxonemal Centriole Elongation}

Tomer Avidor-Reiss, Andrew Ha and Marcus L. Basiri

Cold Spring Harb Perspect Biol 2017; doi: 10.1101/cshperspect.a028142 originally published online January 20, 2017

\section{Subject Collection Cilia}

Ciliary Mechanisms of Cyst Formation in

Polycystic Kidney Disease Ming Ma, Anna-Rachel Gallagher and Stefan Somlo

Photoreceptor Cilia and Retinal Ciliopathies Kinga M. Bujakowska, Qin Liu and Eric A. Pierce

G-Protein-Coupled Receptor Signaling in Cilia Kirk Mykytyn and Candice Askwith

Evolution of Cilia David R. Mitchell

Transition Zone Migration: A Mechanism for Cytoplasmic Ciliogenesis and Postaxonemal Centriole Elongation

Tomer Avidor-Reiss, Andrew Ha and Marcus L. Basiri

Cilia and Obesity Christian Vaisse, Jeremy F. Reiter and Nicolas F. Berbari

\section{Posttranslational Modifications of Tubulin and} Cilia

Dorota Wloga, Ewa Joachimiak, Panagiota Louka, et al.
Cilia in Left-Right Symmetry Breaking

Kyosuke Shinohara and Hiroshi Hamada

Discovery, Diagnosis, and Etiology of Craniofacial

Ciliopathies

Elizabeth N. Schock and Samantha A. Brugmann

Axoneme Structure from Motile Cilia Takashi Ishikawa

Cilia and Ciliopathies in Congenital Heart Disease Nikolai T. Klena, Brian C. Gibbs and Cecilia W. Lo

Sperm Sensory Signaling Dagmar Wachten, Jan F. Jikeli and U. Benjamin Kaupp

Primary Cilia and Coordination of Receptor Tyrosine Kinase (RTK) and Transforming Growth Factor $\beta$ (TGF- $\beta$ ) Signaling

Soren T. Christensen, Stine K. Morthorst, Johanne $B$. Mogensen, et al.

Primary Cilia and Mammalian Hedgehog Signaling Fiona Bangs and Kathryn V. Anderson

For additional articles in this collection, see http://cshperspectives.cshlp.org/cgi/collection/

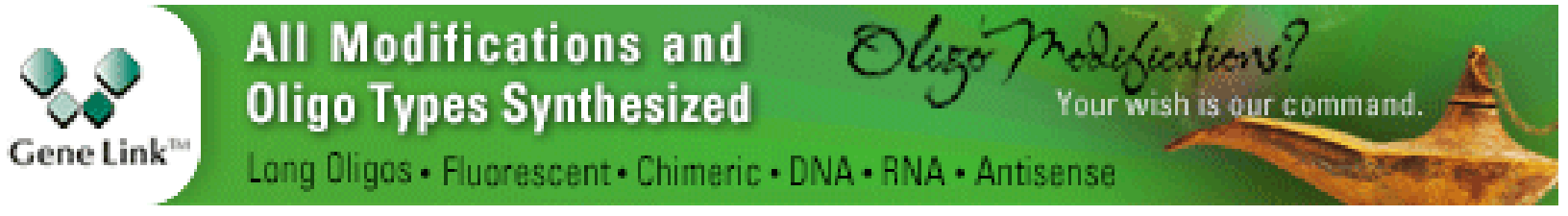


Radial Spokes--A Snapshot of the Motility Regulation, Assembly, and Evolution of Cilia and Flagella

Xiaoyan Zhu, Yi Liu and Pinfen Yang
Cilia and Mucociliary Clearance

Ximena M. Bustamante-Marin and Lawrence E. Ostrowski

For additional articles in this collection, see http://cshperspectives.cshlp.org/cgi/collection/

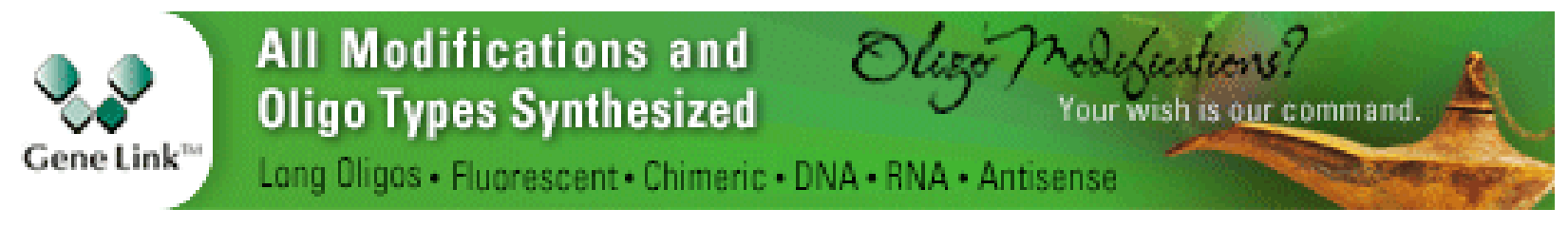

Copyright @ 2017 Cold Spring Harbor Laboratory Press; all rights reserved 